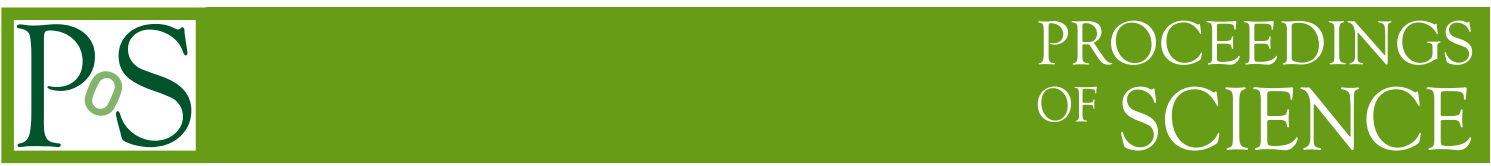

\title{
Production of $\eta_{c}(1 S, 2 S)$ in $e^{+} e^{-}$and $p p$ collisions
}

\section{Izabela Babiarz, ${ }^{a, *}$ Wolfgang Schäfer ${ }^{a}$ and Antoni Szczurek ${ }^{a}$}

${ }^{a}$ Institute of Nuclear Physics Polish Academy of Sciences, PL-31342 Krakow

E-mail: Izabela.Babiarz@ifj.edu.pl, Wolfgang.Schafer@ifj.edu.pl, Antoni.Szczurek@ifj.edu.pl

We derive the light-front wave function (LFWF) representation of the $\gamma^{*} \gamma^{*} \rightarrow \eta_{c}(1 S), \eta_{c}(2 S)$ transition form factor $F\left(Q_{1}^{2}, Q_{2}^{2}\right)$ for two virtual photons in the initial state. For the LFWF, we use different models obtained from the solution of the Schrödinger equation for a variety of $c \bar{c}$ potentials. We compare our results to the BaBar experimental data for the $\eta_{c}(1 S)$ transition form factor, for one real and one virtual photon. We observe that the onset of the asymptotic behaviour is strongly delayed and discuss applicability of the collinear and/or massless limit. In addition, we present a thorough analysis of $\eta_{c}(1 S, 2 S)$ quarkonia hadroproduction in $k_{\perp}$-factorisation in the framework of the light-front potential approach for the quarkonium wave function. The off-shell matrix elements for the $g g \rightarrow \eta_{c}(1 S, 2 S)$ vertices are derived. We discuss the importance of taking into account the gluon virtualities. We present the transverse momentum distributions of $\eta_{c}$ for several models of the unintegrated gluon distributions. Our calculations are performed for four distinct parameterisations for the $c \bar{c}$ interaction potential consistent with the meson spectra. We compare our results for $\eta_{c}(1 S)$ to measurements by the LHCb collaboration and present predictions for $\eta_{c}(2 S)$ production

40th International Conference on High Energy physics - ICHEP2020

July 28 - August 6, 2020

Prague, Czech Republic (virtual meeting)

${ }^{*}$ Speaker 


\section{Introduction: Description of the mechanism $\gamma^{*} \gamma^{*} \rightarrow \eta_{c}(1 S, 2 S)$}

Complementary information for meson structure in quantum chromodynamics can be provided by the study of electromagnetic form factors as well as meson-photon transition form factors. Over a span of several years the attention has been paid mostly on the case of light pseudoscalar meson-photon transition form factors e.g. $\eta, \eta^{\prime}, \pi^{0}$. Similar analysis have been performed for $\eta_{c}$ production. The mass of the $\eta_{c}$ assure a hard scale and validate the perturbative approach even for zero virtuality. Here we will focus on calculating transition form factor for both virtual photon in the light-front frame. For illustration in Fig. 1 we present the generic diagram for applicable process and the scheme of the particle momenta.
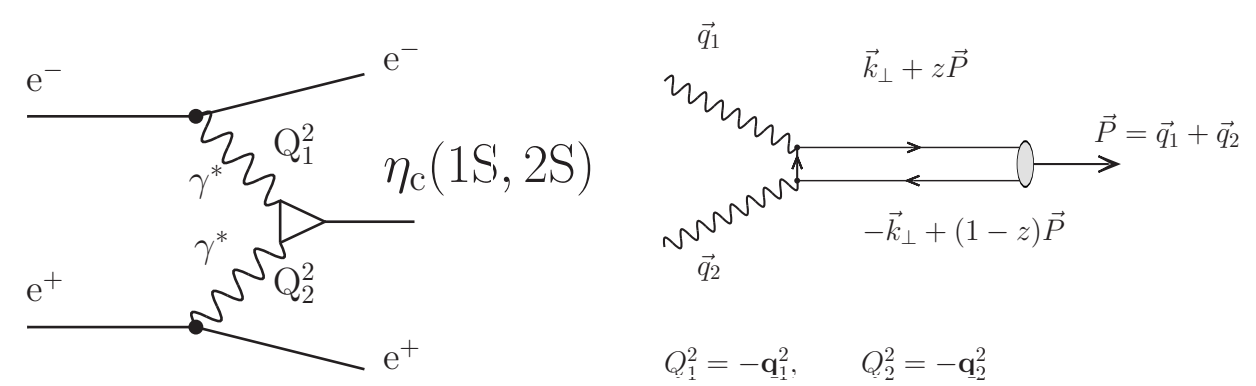

Figure 1: Generic diagram for $\eta_{c}(1 S)$ or the first excited state $\eta_{c}(2 S)$ production. On the right hand side the scheme of the applied momenta.

The general form of the photon-photon fusion amplitude reads:

$$
\mathcal{M}_{\mu \nu}\left(\gamma^{*}\left(q_{1}\right) \gamma^{*}\left(q_{2}\right) \rightarrow \eta_{c}\right)=4 \pi \alpha_{\mathrm{em}}(-i) \varepsilon_{\mu \nu \alpha \beta} q_{1}^{\alpha} q_{2}^{\beta} F\left(Q_{1}^{2}, Q_{2}^{2}\right)
$$

where $Q_{1}^{2}, Q_{2}^{2}$ are photon virtualities and light-front representation of the transition form factor:

$$
\begin{aligned}
F\left(Q_{1}^{2}, Q_{2}^{2}\right)= & e_{c}^{2} \sqrt{N_{c}} 4 m_{c} \cdot \int \frac{d z d^{2} \mathbf{k}}{z(1-z) 16 \pi^{3}} \psi(z, \mathbf{k}) \\
& \left\{\frac{1-z}{\left(\mathbf{k}-(1-z) \mathbf{q}_{2}\right)^{2}+z(1-z) \mathbf{q}_{1}^{2}+m_{c}^{2}}+\frac{z}{\left(\mathbf{k}+z \mathbf{q}_{2}\right)^{2}+z(1-z) \mathbf{q}_{1}^{2}+m_{c}^{2}}\right\} .
\end{aligned}
$$

In order to construct the form factor $F\left(Q_{1}^{2}, Q_{2}^{2}\right)$, we have used light-front wave functions $\psi(z, \boldsymbol{k})$ (see Fig. 2). This wave functions are obtained in few steps, the first step is to solve the Schrödinger equation for several model of $c \bar{c}$ potential and than obtained non-relativistic radial space wave function $u(r)$ are transformed to momentum space $u(p)$ (for more details see [1,2]). Due to Terentev prescription: $\mathbf{p}=\mathbf{k}, p_{z}=(z-1 / 2) M_{c \bar{c}}$ valid for weakly bound system we can rewrite:

$$
\Psi_{\lambda \bar{\lambda}}(z, \mathbf{k})=\overline{\mathrm{U}}_{\lambda}\left(z P_{+}, \mathbf{k}\right) \gamma_{5} \mathrm{v}_{\bar{\lambda}}\left((1-z) P_{+},-\mathbf{k}\right) \psi(z, \mathbf{k}), \quad \psi(z, \mathbf{k})=\frac{\pi}{\sqrt{2 M_{c \bar{c}}}} \frac{u(p)}{p}
$$

It is worth to notice that we treat $\eta_{c}$ meson as a bound state of $c \bar{c}$ and therefore we assume that dominant component in the Fock-state expansion comes from $c \bar{c}$ : 

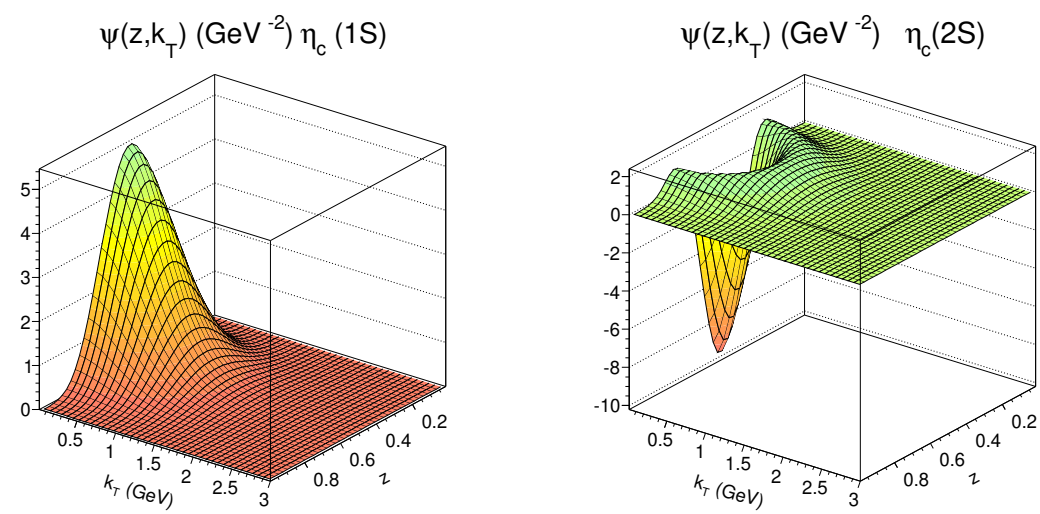

Figure 2: Radial light-front wave function obtained for Buchmüller-Tye potential.

$$
\left|\eta_{c} ; P_{+}, \mathbf{P}\right\rangle=\sum_{i, j, \lambda, \bar{\lambda}} \frac{\delta_{j}^{i}}{\sqrt{N_{c}}} \int \frac{d z d^{2} \mathbf{k}}{z(1-z) 16 \pi^{3}} \Psi_{\lambda \bar{\lambda}}(z, \mathbf{k})\left|c_{i \lambda}\left(z P_{+}, \mathbf{p}_{c}\right) \bar{c}_{\bar{\lambda}}^{j}\left((1-z) P_{+}, \mathbf{p}_{\bar{c}}\right)\right\rangle+\ldots
$$

We vary our numerical results by analysing several model of interaction potential from literature. In the Fig. 3 we present normalized form factor $F\left(Q^{2}, 0\right) / F(0,0)$ for one real and one virtual photon compared to BABAR data. The description of the experimental data appears to be related not only to applied potential model, but also to lower value of $\mathrm{c}$ - quark mass $m_{c}$.
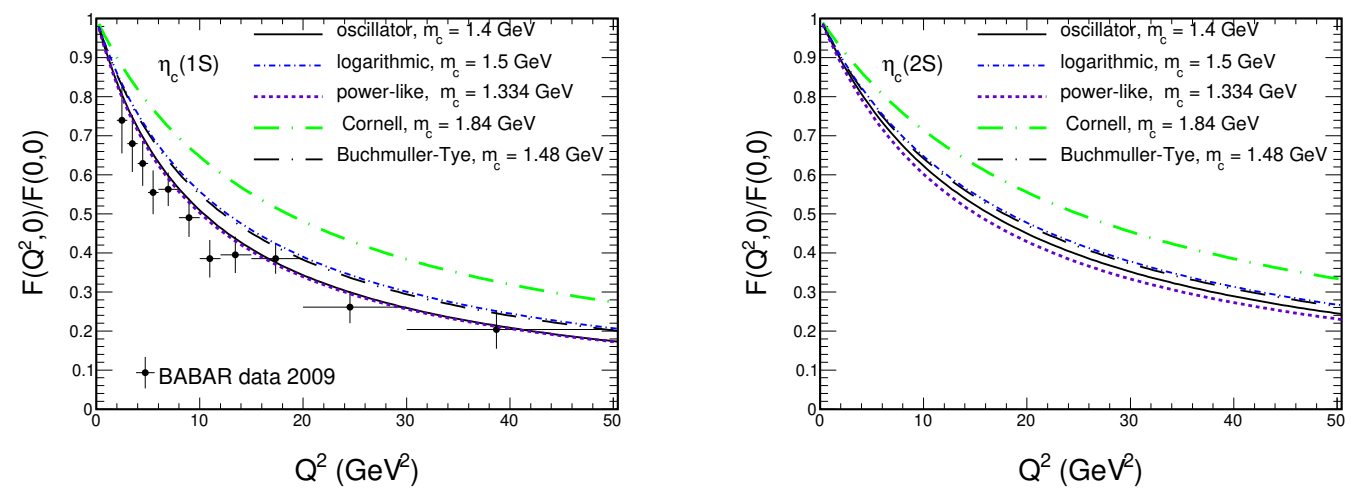

Figure 3: Normalized transition form factor $F\left(Q^{2}, 0\right) / F(0,0)$ as a function of photon virtuality $Q^{2}$. The BaBar data are shown for comparison [3].

\section{Production of $\eta_{c}(1 \mathrm{~S}, 2 \mathrm{~S})$ in $\mathrm{pp}$ collisions}

We applied [4] LF form-factor in $p p$ collision by adjusting quantum numbers to gluon-gluon process. In the $k_{T}$-factorization approach, gluons are off-shell, $q_{i}^{2}=-\boldsymbol{q}_{i}^{2}$ and their four momenta:

$$
q_{1}=\left(q_{1+}, 0, \boldsymbol{q}_{1}\right), q_{2}=\left(0, q_{2-}, \boldsymbol{q}_{2}\right), q_{1+}=x_{1} \sqrt{\frac{s}{2}}, q_{2_{-}}=x_{2} \sqrt{\frac{s}{2}}
$$


We can write the cross section for inclusive $\eta_{c}(1 S)$ or $\eta_{c}(2 S)$ production in the form:

$d \sigma=\int \frac{d x_{1}}{x_{1}} \int \frac{d^{2} \boldsymbol{q}_{1}}{\pi \boldsymbol{q}_{1}^{2}} \mathcal{F}\left(x_{1}, \boldsymbol{q}_{1}^{2}, \mu_{F}^{2}\right) \int \frac{d x_{2}}{x_{2}} \int \frac{d^{2} \boldsymbol{q}_{2}}{\pi \boldsymbol{q}_{2}^{2}} \mathcal{F}\left(x_{2}, \boldsymbol{q}_{2}^{2}, \mu_{F}^{2}\right) \frac{1}{2 x_{1} x_{2} s} \overline{|\mathcal{M}|^{2}} d \Phi(2 \rightarrow 1)$,

where the phase space element: $d \Phi(2 \rightarrow 1)=(2 \pi)^{4} \delta^{(4)}\left(q_{1}+q_{2}-p\right) \frac{d^{4} p}{(2 \pi)^{3}} \delta\left(p^{2}-M_{\eta_{c}}^{2}\right)$.

$$
\mathcal{M}^{a b}=\frac{q_{1 \perp}^{\mu} q_{2 \perp}^{v}}{\left|\boldsymbol{q}_{1}\right|\left|\boldsymbol{q}_{2}\right|} \mathcal{M}_{\mu \nu}^{a b}=\frac{q_{1+} q_{2-}}{\left|\boldsymbol{q}_{1}\right|\left|\boldsymbol{q}_{2}\right|} n^{+\mu} n^{-v} \mathcal{M}_{\mu \nu}^{a b}=\frac{x_{1} x_{2} s}{2\left|\boldsymbol{q}_{1}\right|\left|\boldsymbol{q}_{2}\right|} n^{+\mu} n^{-v} \mathcal{M}_{\mu \nu}^{a b}
$$

Therefore, explicitly matrix element: $n^{+\mu} n^{-\mu} \mathcal{M}_{\mu \nu}^{a b}=4 \pi \alpha_{s}(-i)\left[\boldsymbol{q}_{1}, \boldsymbol{q}_{2}\right] \operatorname{Tr}\left[t^{a} t^{b}\right] / \sqrt{N_{c}} I\left(\boldsymbol{q}_{1}^{2}, \boldsymbol{q}_{2}^{2}\right)$ and averaging over colors, we obtain our final result:

$$
\begin{aligned}
\frac{d \sigma}{d y d^{2} \boldsymbol{p}}=\int \frac{d^{2} \boldsymbol{q}_{1}}{\pi \boldsymbol{q}_{1}^{4}} & \mathcal{F}\left(x_{1}, \boldsymbol{q}_{1}^{2}\right) \int \frac{d^{2} \boldsymbol{q}_{2}}{\pi \boldsymbol{q}_{2}^{4}} \mathcal{F}\left(x_{2}, \boldsymbol{q}_{2}^{2}\right) \delta^{(2)}\left(\boldsymbol{q}_{1}+\boldsymbol{q}_{2}-\boldsymbol{p}\right) \\
& \times \frac{\pi^{3} \alpha_{s}^{2}}{N_{c}\left(N_{c}^{2}-1\right)}|| \boldsymbol{q}_{1}|| \boldsymbol{q}_{2}\left|\sin \left(\phi_{1}-\phi_{2}\right) I\left(\boldsymbol{q}_{1}^{2}, \boldsymbol{q}_{2}^{2}\right)\right|^{2} .
\end{aligned}
$$

The relation $I\left(\boldsymbol{q}_{1}^{2}, \boldsymbol{q}_{2}^{2}\right)$ with the form factor $\gamma^{*} \gamma^{*} \rightarrow \eta_{c}: F\left(Q_{1}^{2}, Q_{2}^{2}\right)=e_{c}^{2} \sqrt{N_{c}} I\left(\boldsymbol{q}_{1}^{2}, \boldsymbol{q}_{2}^{2}\right)$, and going to in NRQCD limit the transition form factor takes the form:

$$
F_{\mathrm{NRQCD}}\left(Q_{1}^{2}, Q_{2}^{2}\right)=\frac{4 e_{c}^{2} \sqrt{N_{c}}}{\sqrt{\pi M_{\eta_{c}}}} \frac{1}{M_{\eta_{c}}^{2}+Q_{1}^{2}+Q_{2}^{2}} R(0),
$$

where $R(0)$ is radial wave function of the potential-model at the spatial origin.

The normalization of the cross section crucially depends on the value of the form factor at on-shell point, thus we extract $F(0,0)$ from experimental value of radiative decay width (see Table 1) in leading order:

$$
\Gamma_{\mathrm{LO}}\left(\eta_{c} \rightarrow \gamma \gamma\right)=\frac{\pi}{4} \alpha_{\mathrm{em}}^{2} M_{\eta_{c}}^{3}|F(0,0)|^{2}
$$

and the expressions for the widths at Next Leading Order according to Ref. [5]:

$$
\Gamma_{\mathrm{NLO}}\left(\eta_{c} \rightarrow \gamma \gamma\right)=\Gamma_{\mathrm{LO}}\left(\eta_{c} \rightarrow \gamma \gamma\right)\left(1-\frac{20-\pi^{2}}{3} \frac{\alpha_{s}}{\pi}\right) .
$$

In Figs. 4 and 5 we present differential cross section as a function of transverse momentum for prompt $\eta_{c}(1 S)$ and $\eta_{c}(2 S)$ production compared with the LHCb data for $\sqrt{s}=7,8 \mathrm{TeV}$ [7] and preliminary experimental data for $\sqrt{s}=13 \mathrm{TeV}$ [8] for the interval in rapidity $2.0<y<4.5$. In the numerical calculation we used several unintegrated gluon distributions and we applied form factor calculated from the power-law potential as explained in the section 1. Whereas in the Fig. 6 we put in an appearance transverse momentum distribution of the $\eta_{c}(1 S)$ (left panel) and $\eta_{c}(2 S)$ (middle panel) with form factor obtained from wave functions for different potential model and the same normalization at the on shell point of the form factor. 


\begin{tabular}{c|c|c|c}
\hline \hline & $\begin{array}{c}\text { Experimental values } \\
\Gamma_{\gamma \gamma}(\mathrm{keV})[6]\end{array}$ & $\begin{array}{c}\text { Derived from LO } \\
|F(0,0)|\left[\mathrm{GeV}^{-1}\right]\end{array}$ & $\begin{array}{c}\text { Derived from NLO } \\
|F(0,0)|_{\gamma \gamma}\left[\mathrm{GeV}^{-1}\right]\end{array}$ \\
\hline$\eta_{c}(1 S)$ & $5.0 \pm 0.4$ & $0.067 \pm 0.003$ & $0.079 \pm 0.003$ \\
$\eta_{c}(2 S)$ & $1.9 \pm 1.3 \cdot 10^{-4} \cdot \Gamma_{\eta_{c}(2 S)}$ & $0.033 \pm 0.012$ & $0.038 \pm 0.014$ \\
\hline \hline
\end{tabular}

Table 1: Radiative decay widths as well as $|F(0,0)|$ obtained from $\Gamma_{\gamma \gamma}$ using leading order and next-toleading order approximation.
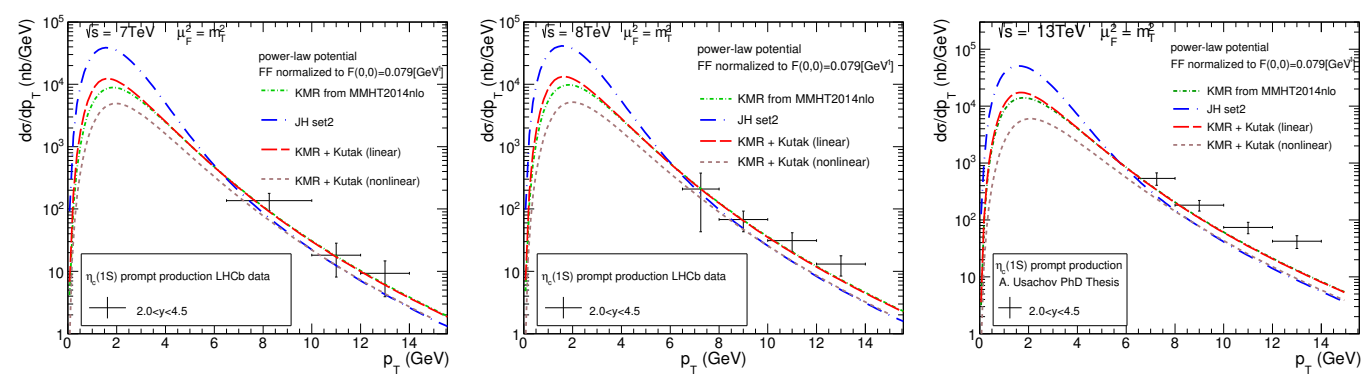

Figure 4: Differential cross section as a function of transverse momentum for prompt $\eta_{c}(1 S)$ production compared with the LHCb data for $\sqrt{s}=7,8 \mathrm{TeV}$ [7] [8].
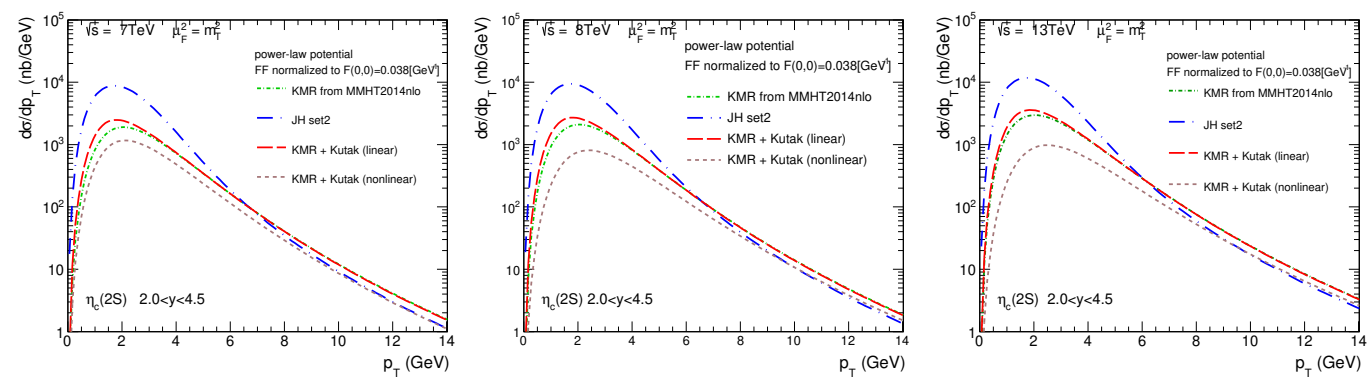

Figure 5: Differential cross section as a function of transverse momentum for prompt $\eta_{c}(2 S)$ production for $\sqrt{s}=7,8,13 \mathrm{TeV}$.

On the right panel of the Fig. 6 we demonstrate the results with different normalization of the form factor, normalized to experimental value, exact value from light front wave functions and point like form factor. We wish to point out it is important to taking into account gluon virtualities in the $\eta_{c}$ prompt hadroproduction. This kind of processes are also good probe of Unintegrated Gluon Distributions.

\section{Acknowledgement}

This study was partially supported by the Polish National Science Center under Grant No. 2018/31/B/ST2/03537. 

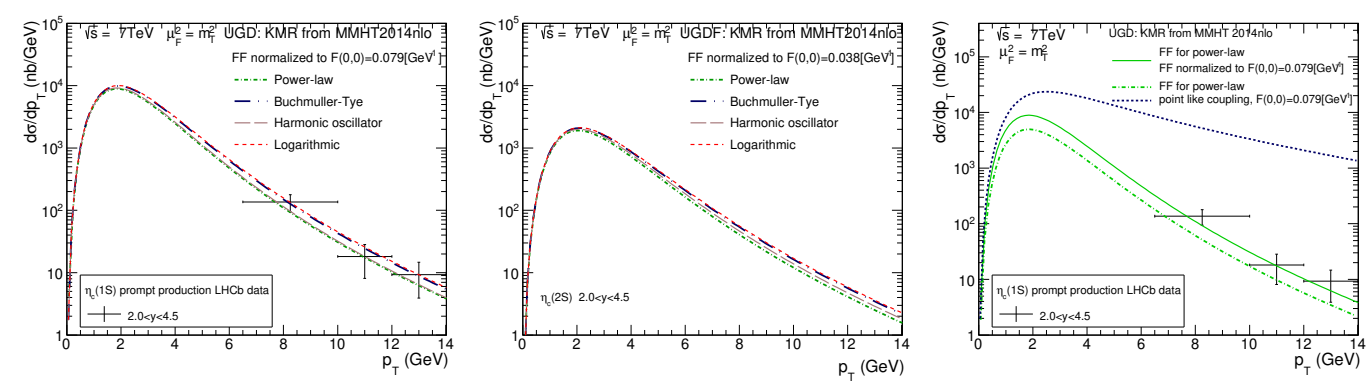

Figure 6: Distribution in transverse momentum of the $\eta_{c}(1 S)$ (left panel) and the $\eta_{c}(2 S)$ (middle panel) for form factor calculated, from different potential model, with the same normalization at the on shell point. On the right panel comparison of the results with different normalization for the form factor for Power-law potential.

\section{References}

[1] I. Babiarz et al., $\gamma^{*} \gamma^{*} \rightarrow \eta_{c}(1 S, 2 S)$ transition form factors for spacelike photons, Phys. Rev. $D 100$ (2019), no.5, 054018 [hep-ph/1908.07802]

[2] J. Cepila et al., Theoretical uncertainties in exclusive electroproduction S-wave heavy quarkonia, Eur. Phys. J. C 79 (2019), no.6, 495 [hep-ph/1901. 02664]

[3] J. P. Lees et al. [BaBar], Measurement of the $\gamma \gamma *-->\eta_{c}$ transition form factor, Phys. Rev. D 81 (2010), 052010 [hep-ex/1002 . 3000]

[4] I. Babiarz et al., Prompt hadroproduction of $\eta_{c}(1 S, 2 S)$ in the $k_{T}$-factorization approach, JHEP 02 (2020) 037 [hep-ph/1911.03403]

[5] J. P. Lansberg et al., Two-photon width of eta(c) and eta(c)-prime from heavy-quark spin symmetry, Phys. Rev. D 74 (2006) 034001 [hep-ph/0603113]

[6] M. Tanabashi et al. [Particle Data Group], Review of Particle Physics, Phys. Rev. D 98 (2018) no.3, 030001

[7] R. Aaij et al. [LHCb], Measurement of the $\eta_{c}(1 S)$ production cross-section in protonproton collisions via the decay $\eta_{c}(1 S) \rightarrow$ p, Eur. Phys. J. C 75 (2015) no.7, 311 [hep-ex/1409.3612]

[8] A. Usachov, Study of charmonium production using decays to hadronic final states with the LHCb experiment, [hep-ex/1910 . 08796] 